\title{
Fixed point theorems of nondecreasing order-Ćirić-Lipschitz mappings in normed vector spaces without normalities of cones
}

\author{
Zhilong Li ${ }^{\mathrm{a}, \mathrm{b}}$, Shujun Jiang ${ }^{\mathrm{c}, *}$ \\ a School of Statistics, Jiangxi University of Finance and Economics, Nanchang, 330013, China. \\ ${ }^{b}$ Research Center of Applied Statistics, Jiangxi University of Finance and Economics, Nanchang, 330013, China. \\ ${ }^{c}$ Department of Mathematics, Jiangxi University of Finance and Economics, Nanchang, 330013, China. \\ Communicated by R. Saadati
}

\begin{abstract}
We introduce the concept of order-Ćirić-Lipschitz mappings, and prove some fixed point theorems for such kind of mappings in normed vector spaces without assuming the normalities of cones by using upper and lower solutions method, which improve many existing results of order-Lipschitz mappings in Banach spaces or Banach algebras. It is worth mentioning that even in the setting of normal cones, the main results in this paper are still new since the sum of spectral radius or the sum of restricted constants may be greater than or equal to 1 . (C)2017 all rights reserved.
\end{abstract}

Keywords: Fixed point, order-Ćirić-Lipschitz mapping, Picard-complete, $w$-complete. 2010 MSC: 06A07, 47H10.

\section{Introduction and preliminaries}

Ćirić's contraction in metric spaces was primitively introduced and studied by Ćirić [3], which generalizes and includes Banach contraction [1], Kannan contraction [7], and Chatterjea contraction [2]. Let $(X, d)$ be a metric space. Recall that a mapping $T: X \rightarrow X$ is called a Ćirić's contraction if there exist nonnegative numbers $q, r, s, t$ with $q+r+s+2 t<1$ such that

$$
d(T x, T y) \leqslant q d(x, y)+r d(x, T x)+s d(y, T y)+t[d(x, T y)+d(y, T x)], \quad \forall x, y \in X .
$$

Note that a Ćirić's contraction must be a Lipschitz mapping. Thus motivated by [3], we introduce the concept of Ćirić order-Lipschitz mapping in topological vector spaces as follows.

Definition 1.1. Let $P$ be a cone of a topological vector space $E, D \subset E$ and $\preceq$ the partial order induced by $P$. A mapping $T: D \rightarrow E$ is called an order-Ćirić-Lipschitz mapping if there exist $A_{i}, B_{i}: P \rightarrow P(i=$ $1,2,3,4,5)$ (or nonnegative real numbers $A_{i}, B_{i}(i=1,2,3,4,5)$ ) such that for each $x, y \in D$ with $y \preceq x$,

$$
\begin{gathered}
-B_{1}(x-y)-B_{2}(x-T x)_{+}-B_{3}(y-T y)_{+}-B_{4}(x-T y)_{+}-B_{5}(y-T x)_{+} \preceq T x-T y \\
\preceq A_{1}(x-y)+A_{2}(x-T x)_{+}+A_{3}(y-T y)_{+}+A_{4}(x-T y)_{+}+A_{5}(y-T x)_{+},
\end{gathered}
$$

\footnotetext{
*Corresponding author

Email addresses: lz1771218@sina.com (Zhilong Li), jiangshujuns@sina.com (Shujun Jiang)
} 
where

$$
u_{+}= \begin{cases}u, & u \in P, \\ -u, & u \in-P, \\ \theta, & \text { o.w. }\end{cases}
$$

for each $u \in E$.

In particular when $A_{i} u \equiv \theta(i=2,3,4,5)$ and $B_{i} u \equiv \theta(i=2,3,4,5)$, the generalized order-Lipschitz mapping is reduced to an order-Lipschitz mapping considered in $[8,10,11]$, where the cone is necessarily assumed to be normal. Recently, without assuming the normality of P, Jiang and Li [6] proved the following fixed point theorem of order-Lipschitz mappings by introducing the concept of Picard-completeness and applying the sandwich theorem in the sense of $w$-convergence.

Theorem 1.2 ([6]). Let $\mathrm{P}$ be a solid cone of a Banach algebra $(\mathrm{E},\|\cdot\|)$ and $\mathrm{u}_{0}, v_{0} \in \mathrm{E}$ with $\mathrm{u}_{0} \preceq v_{0}$, and $\mathrm{T}: \mathrm{D}=\left[\mathrm{u}_{0}, v_{0}\right] \rightarrow \mathrm{E}$ a nondecreasing order-Lipschitz mapping restricted with vectors (i.e., there exist $\mathrm{A}_{1} \in \mathrm{P}$ and $A_{i}=\theta(i=2,3,4,5)$ such that such that (1.1) is satisfied $)$. Assume that $\mathfrak{u}_{0} \preceq T u_{0}, T v_{0} \preceq v_{0}$ (i.e., $\mathfrak{u}_{0}$ and $v_{0}$ are a pair of lower and upper solutions of $\mathrm{T}), \mathrm{r}\left(\mathrm{A}_{1}\right)<1$ and $\mathrm{T}$ is Picard-complete at $\mathrm{u}_{0}$ and $v_{0}$. Then $\mathrm{T}$ has a unique fixed point $x^{*} \in\left[u_{0}, v_{0}\right]$, and $x_{n} \stackrel{w}{\rightarrow} x^{*}$ for each $x_{0} \in\left[u_{0}, v_{0}\right]$, where $\left\{x_{n}\right\}=\mathrm{O}\left(T, x_{0}\right)$ and $\mathrm{O}\left(\mathrm{T}, \mathrm{x}_{0}\right)$ denotes the Picard iteration sequence of $\mathrm{T}$ at $\mathrm{x}_{0}$, i.e., $\mathrm{x}_{\mathrm{n}}=\mathrm{T}^{\mathrm{n}} \mathrm{x}_{0}$ for each $\mathrm{n}$.

In this paper, we shall prove some new fixed point theorems of order-Ćirić-Lipschitz mappings in normed vector spaces without assuming the normalities of cones by using upper and lower solutions method, which improve many existing results of order-Lipschitz mappings in Banach spaces or Banach algebras. It is worth mentioning that even in the setting of normal cones, the main results in this paper are still new since the sum of spectral radius or the sum of restricted constants may be greater than or equal to 1 .

Let $E$ be a topological vector space. A nonempty closed subset $P$ of $E$ is a cone if it is such that $a x+b y \in P$, for each $x, y \in P$ and each $a, b \geqslant 0$, and $P \cap(-P)=\{\theta\}$, where $\theta$ is the zero element of $E$. Each cone $P$ of $E$ determines a partial order $\preceq$ on $E$ by $x \preceq y \Leftrightarrow y-x \in P$ for each $x, y \in X$. For each $u_{0}, v_{0} \in E$ with $u_{0} \preceq v_{0}$, we set $\left[u_{0}, v_{0}\right]=\left\{u \in E: u_{0} \preceq u \preceq v_{0}\right\},\left[u_{0},+\infty\right)=\left\{x \in E: u_{0} \preceq x\right\}$ and $\left(-\infty, v_{0}\right]=\left\{x \in E: x \preceq v_{0}\right\}$. A cone $P$ of $E$ is solid [4] if $\operatorname{int}(P)$ is nonempty, where $\operatorname{int}(P)$ denotes the interior of $P$. For each $x, y \in E$ with $y-x \in \operatorname{int}(P)$, we write $x \ll y$.

Let $(E,\|\cdot\|)$ be a normed vector space. A cone $P$ of $E$ is normal [4] if there is a positive real number $N$ such that $x, y \in E$ and $\theta \preceq x \preceq y$ implies that $\|x\| \leqslant N\|y\|$, and the minimal $N$ is called a normal constant of $P$. Note that if $P$ is non-normal then the sandwich theorem does not hold in the sense of norm-convergence.

Definition 1.3. Let $P$ be a solid cone of a topological space $E,\left\{x_{n}\right\} \subset E$ and $D \subset E$.

(i) The sequence $\left\{x_{n}\right\}$ is $w$-convergent $[5,9]$ if for each $\epsilon \in \operatorname{int}(P)$, there is a positive integer $n_{0}$ and $x \in E$ such that $x-\epsilon \ll x_{n} \ll x+\epsilon$ for each $n \geqslant n_{0}$ (denote $x_{n} \stackrel{w}{\rightarrow} x$ and $x$ is called a $w$-limit of $\left.\left\{x_{n}\right\}\right)$.

(ii) The sequence $\left\{x_{n}\right\}$ is $w$-Cauchy [6] if for each $\epsilon \in \operatorname{int}(P)$, there is a positive integer $n_{0}$ such that $-\epsilon \ll x_{n}-x_{m} \ll \epsilon$ for each $m, n \geqslant n_{0}$, i.e., $x_{n}-x_{m} \stackrel{w}{\rightarrow} \theta(m, n \rightarrow \infty)$.

(iii) The space $E$ is $w$-complete if each $w$-Cauchy sequence in $E$ is $w$-convergent;

(iv) The subset $D$ is $w$-closed [6] if for each $\left\{x_{n}\right\} \subset D, x_{n} \stackrel{w}{\rightarrow} x$ implies $x \in D$.

Lemma 1.4 ([6,9]). Let $\mathrm{P}$ be a solid cone of a normed vector space $(\mathrm{E},\|\cdot\|)$ and $\mathfrak{u}_{0}, v_{0} \in \mathrm{E}$ with $\mathrm{u}_{0} \preceq v_{0}$. Then

(i) each sequence $\left\{x_{n}\right\} \subset E$ has a unique w-limit; 
(ii) the partial order intervals $\left[\mathrm{u}_{0}, v_{0}\right],\left[\mathrm{u}_{0},+\infty\right)$ and $\left(-\infty, v_{0}\right]$ are $w$-closed;

(iii) for each $\left\{x_{n}\right\},\left\{y_{n}\right\},\left\{z_{n}\right\} \subset E$ with $x_{n} \preceq y_{n} \preceq z_{n}$ for each $n, x_{n} \stackrel{w}{\rightarrow} z$ and $z_{n} \stackrel{w}{\rightarrow} z$ imply $y_{n} \stackrel{w}{\rightarrow} z$, where $z \in \mathrm{E}$.

Lemma $1.5([5,9])$. Let $\mathrm{P}$ be a solid cone of a normed vector space $(\mathrm{E},\|\cdot\|)$ and $\mathrm{x}_{\mathrm{n}} \subset \mathrm{E}$. Then $\mathrm{x}_{\mathrm{n}} \stackrel{\|\cdot\|}{\rightarrow} \mathrm{x}$ implies $\mathrm{x}_{\mathrm{n}} \stackrel{w}{\rightarrow} \mathrm{x}$. Moreover, if $\mathrm{P}$ is normal then $\mathrm{x}_{\mathrm{n}} \stackrel{w}{\rightarrow} \mathrm{x} \Leftrightarrow \mathrm{x}_{\mathrm{n}} \stackrel{\|\cdot\|}{\rightarrow} \mathrm{x}$.

Proposition 1.6. Let $\mathrm{P}$ be a normal solid cone of a normed vector space $(\mathrm{E},\|\cdot\|)$. Then $\mathrm{E}$ is $w$-complete if and only if it is complete.

Proof. Suppose that $E$ is complete and $\left\{x_{n}\right\}$ is a $w$-Cauchy sequence of $E$. By Lemma 1.5 and the normality of $P,\left\{x_{n}\right\}$ is a Cauchy sequence of $E$, and hence it is convergent by the completeness of $E$. Moreover by Lemma 1.5, $\left\{x_{n}\right\}$ is $w$-convergent. This shows $E$ is $w$-complete.

Suppose that $E$ is $w$-complete and $\left\{x_{n}\right\}$ is a Cauchy sequence of $E$. By Lemma 1.5, $\left\{x_{n}\right\}$ is a $w$-Cauchy sequence of $E$, and hence it is convergent by the $w$-completeness of $E$. Moreover by Lemma 1.5 and the normality of $P,\left\{x_{n}\right\}$ is convergent. This shows $E$ is complete.

Definition 1.7 ([6]). Let $P$ be a solid cone of a topological vector space $(E,\|\cdot\|), D \subset E, x_{0} \in D$ and $T: D \rightarrow E$. If the Picard iteration sequence $O\left(T, x_{0}\right)$ is $w$-convergent provided that it is $w$-Cauchy, then $T$ is said to be Picard-complete at $x_{0}$. Moreover, if $T$ is Picard-complete at each $x \in D$, then $T$ is said to be Picard-complete on D.

Remark 1.8. If $\mathrm{E}$ is a $w$-complete topological vector space then the mapping $\mathrm{T}: \mathrm{E} \rightarrow \mathrm{E}$ is Picard-complete on $E$. Consequently, if $P$ is a normal solid cone of a Banach space $(E,\|\cdot\|)$ then each mapping $T: E \rightarrow E$ is Picard-complete on E by Proposition 1.6.

Definition 1.9. Let $P$ be a solid cone of a topological vector space $E$ and $D \subset E$. A mapping $T: D \rightarrow E$ is $w$-continuous at $x_{0} \in E$ if for each $\left\{x_{n}\right\} \subset E, x_{n} \stackrel{w}{\rightarrow} x_{0}$ implies $T x_{n} \stackrel{w}{\rightarrow} T x_{0}$. If $T$ is $w$-continuous at each $x \in \mathrm{D}$, then $\mathrm{T}$ is $w$-continuous on $\mathrm{D}$.

Lemma 1.10. Let $\mathrm{P}$ be a solid cone of a normed vector space $(\mathrm{E},\|\cdot\|)$ and $\mathrm{A}: \mathrm{E} \rightarrow \mathrm{E}$ a linear bounded mapping with $\mathrm{A}(\mathrm{P}) \subset \mathrm{P}$. Then $\mathrm{A}$ is $w$-continuous on $\mathrm{E}$.

Proof. Let $x \in E$ and $\left\{x_{n}\right\}$ be a sequence in $E$ such that $x_{n} \stackrel{w}{\rightarrow} x$. For each $\epsilon \in \operatorname{int}(P)$, it is clear that $\frac{\epsilon}{m} \in \operatorname{int}(P)$ for each $m$, and hence there exists $n_{m}$ such that $-\frac{\epsilon}{m} \ll x_{n}-x \ll \frac{\epsilon}{m}$ for each $n \geqslant n_{m}$. Note that $A$ is a linear mapping with $A(P) \subset P$, then $-\frac{A \epsilon}{m} \preceq A x_{n}-A x \preceq \frac{A \epsilon}{m}$ for each $n \geqslant n_{m}$. It is clear that $\frac{A \epsilon}{\mathrm{m}} \stackrel{\|\cdot\|}{\rightarrow} \theta(\mathrm{m} \rightarrow \infty)$ since $A$ is bounded, and hence $\frac{A \epsilon}{\mathrm{m}} \stackrel{w}{\rightarrow} \theta(\mathrm{m} \rightarrow \infty)$ by Lemma 1.5. Moreover, by Lemma 1.4 (iii), we obtain $A x_{n}-A x \stackrel{w}{\rightarrow} \theta$, i.e., $A$ is continuous at $x$.

\section{Main results}

In this section, we first consider the existence of fixed points of order-Ćirić-Lipschitz mappings.

Theorem 2.1. Let $\mathrm{P}$ be a solid cone of a normed vector space $(\mathrm{E},\|\cdot\|), \mathrm{x}_{0} \in \mathrm{E}$ and $\mathrm{T}: \mathrm{D}=\left[\mathrm{x}_{0},+\infty\right) \rightarrow \mathrm{E} a$ nondecreasing order-Ćirić-Lipschitz mapping restricted with linear bounded mappings $A_{i}: P \rightarrow P(i=1,2,3,4,5)$. Assume that $\mathrm{x}_{0} \preceq \mathrm{T} \mathrm{x}_{0}, \mathrm{~T}$ is Picard-complete at $\mathrm{x}_{0}$ and

$$
r\left(A_{2}+A_{5}\right)<1, r\left(\left(I-A_{2}-A_{5}\right)^{-1}\left(A_{1}+A_{3}+A_{5}\right)\right)<1 .
$$

Then $\mathrm{T}$ has a fixed point in $\left[\mathrm{x}_{0},+\infty\right)$. 
Proof. Let $\mathrm{B}: \mathrm{P} \rightarrow \mathrm{P}$ be an arbitrary linear bounded mapping with $\mathrm{r}(\mathrm{B})<1$, then $\mathrm{I}-\mathrm{B}$ is invertible, denote the inverse of $\mathrm{I}-\mathrm{B}$ by $(\mathrm{I}-\mathrm{B})^{-1}$. Moreover, it follows from Neumann's formula that

$$
(I-B)^{-1}=\sum_{n=0}^{\infty} B^{n}=I+B+B^{2}+\cdots+B^{n}+\cdots,
$$

which implies that $(\mathrm{I}-\mathrm{B})^{-1}: \mathrm{P} \rightarrow \mathrm{P}$ is a linear bounded mapping. It follows from $\mathrm{r}(\mathrm{B})<1$ and Gelfand's formula that there exist a positive integer $n_{1}$ and $\beta \in(r(B), 1)$ such that

$$
\left\|B^{n}\right\| \leqslant \beta^{n}, \quad \forall n \geqslant n_{1} .
$$

Thus for each $u \in P$, we get

$$
\left\|B^{n} u\right\| \leqslant\left\|B^{n}\right\|\left\|u \mid \leqslant \beta^{n}\right\| u \|, \quad \forall n \geqslant n_{1},
$$

which implies $\mathrm{B}^{\mathrm{n}} \mathrm{u} \stackrel{\|\cdot\|}{\rightarrow} \theta$ for each $u \in P$, and hence by Lemma 1.5,

$$
\mathrm{B}^{\mathrm{n}} \mathrm{u} \stackrel{w}{\rightarrow} \theta, \quad \forall \mathrm{u} \in \mathrm{P} .
$$

Let $x_{n}=T^{n} x_{0}$ for each $n$. Note that $T$ is nondecreasing on $\left[x_{0},+\infty\right)$, then it follows from $x_{0} \preceq T x_{0}$ that

$$
x_{0} \preceq x_{1} \preceq \cdots \preceq x_{n} \preceq x_{n+1}, \quad \forall n .
$$

By (1.1) and (2.4),

$$
\begin{aligned}
\theta & \preceq x_{n+1}-x_{n}=T x_{n}-T x_{n-1} \\
& \preceq A_{1}\left(x_{n}-x_{n-1}\right)+A_{2}\left(x_{n+1}-x_{n}\right)+A_{3}\left(x_{n+1}-x_{n}\right)+A_{4}\left(x_{n}-x_{n}\right)+A_{5}\left(x_{n+1}-x_{n-1}\right) \\
& =A_{1}\left(x_{n}-x_{n-1}\right)+A_{2}\left(x_{n+1}-x_{n}\right)+A_{3}\left(x_{n}-x_{n-1}\right)+A_{5}\left(x_{n+1}-x_{n}\right)+A_{5}\left(x_{n}-x_{n-1}\right), \quad \forall n,
\end{aligned}
$$

and so

$$
\theta \preceq\left(I-A_{2}-A_{5}\right)\left(x_{n+1}-x_{n}\right) \preceq\left(A_{1}+A_{3}+A_{5}\right)\left(x_{n}-x_{n-1}\right), \quad \forall n .
$$

Note that $\left(I-A_{2}-A_{5}\right)^{-1}: P \rightarrow P$ is a linear bounded mapping by $r\left(A_{2}+A_{5}\right)<1$ and taking $B=A_{2}+A_{5}$ in (2.2). Acting (2.5) with $\left(I-A_{2}-A_{5}\right)^{-1}$, we get

$$
\theta \preceq x_{\mathrm{n}+1}-x_{\mathrm{n}} \preceq A\left(x_{\mathrm{n}}-x_{\mathrm{n}-1}\right), \quad \forall \mathrm{n},
$$

where $A=\left(I-A_{2}-A_{5}\right)^{-1}\left(A_{1}+A_{3}+A_{5}\right)$. Clearly, $(I-A)^{-1}$ is also a linear bounded mapping since $r(A)<1$ by (2.1). Taking $B=A$ in (2.2), by (2.6), we have

$$
\begin{aligned}
\theta \preceq x_{m}-x_{n}=\sum_{i=n}^{m-1}\left(x_{i+1}-x_{i}\right) & \preceq \sum_{i=n}^{m-1} A^{i}\left(x_{1}-x_{0}\right) \\
& =A^{n} \sum_{i=0}^{m-1} A^{i}\left(x_{1}-x_{0}\right) \preceq A^{n}(I-A)^{-1}\left(x_{1}-x_{0}\right), \quad \forall m>n .
\end{aligned}
$$

Taking $B=A$ and $u=(I-A)^{-1}\left(x_{1}-x_{0}\right)$ in (2.3), it follows that

$$
A^{n}(I-A)^{-1}\left(x_{1}-x_{0}\right) \stackrel{w}{\rightarrow} \theta(n \rightarrow \infty),
$$

which together with (2.7) and Lemma 1.4 (iii) implies that

$$
x_{m}-x_{n} \stackrel{w}{\rightarrow} \theta(m>n \rightarrow \infty),
$$


i.e., $\left\{x_{n}\right\}$ is a $w$-Cauchy sequence. Thus by the Picard-completeness of $T$ at $x_{0}$, there exists $x^{*} \in E$ such that

$$
x_{\mathrm{n}} \stackrel{w}{\rightarrow} x^{*} .
$$

It is clear that $\left[x_{n},+\infty\right)$ is $w$-closed for each $n$ by Lemma 1.4 (ii), and so it follows from (2.4) and (2.9) that

$$
x_{\mathrm{n}} \preceq x^{*}, \mathrm{n}=0,1,2, \cdots .
$$

Moreover, by the nondecreasing property of $\mathrm{T}$ on $\left[\mathrm{x}_{0},+\infty\right)$,

$$
x_{n+1}=T x_{n} \preceq T x^{*}, n=0,1,2, \cdots,
$$

which together with (2.9) and Lemma 1.4 (ii) implies that

$$
x^{*} \preceq T x^{*} .
$$

By (1.1), (2.4), (2.10), and (2),

$$
\begin{aligned}
\theta & \preceq T x^{*}-x_{n+1} \\
& =T x^{*}-T x_{n} \\
& \preceq A_{1}\left(x^{*}-x_{n}\right)+A_{2}\left(T x^{*}-x^{*}\right)+A_{3}\left(x_{n+1}-x_{n}\right)+A_{4}\left(x^{*}-x_{n+1}\right)+A_{5}\left(T x^{*}-x_{n}\right), \quad \forall n .
\end{aligned}
$$

Letting $n \rightarrow \infty$ in (2.12), by (2.8), (2.9), Lemma 1.4 (iii), and Lemma 1.10 we get

$$
\theta \preceq T x^{*}-x^{*} \preceq A_{2}\left(T x^{*}-x^{*}\right)+A_{5}\left(T x^{*}-x^{*}\right),
$$

and so

$$
\left(I-A_{2}-A_{5}\right)\left(T x^{*}-x^{*}\right) \preceq \theta .
$$

Acting the above inequality with $\left(I-A_{2}-A_{5}\right)^{-1}$, we have $\theta \preceq T x^{*}-\chi^{*} \preceq \theta$, i.e., $x^{*}=T x^{*}$.

Theorem 2.2. Let $\mathrm{P}$ be a solid cone of a normed vector space $(\mathrm{E},\|\cdot\|), \mathrm{y}_{0} \in \mathrm{E}$ and $\mathrm{T}: \mathrm{D}=\left(-\infty, \mathrm{y}_{0}\right] \rightarrow \mathrm{E} a$ nondecreasing order-Ćirić-Lipschitz mapping restricted with linear bounded mappings $A_{i}: P \rightarrow P(i=1,2,3,4,5)$. Assume that $\mathrm{T} \mathrm{y}_{0} \preceq \mathrm{y}_{0}, \mathrm{~T}$ is Picard-complete at $\mathrm{y}_{0}$ and

$$
r\left(A_{3}+A_{4}\right)<1, r\left(\left(I-A_{3}-A_{4}\right)^{-1}\left(A_{1}+A_{2}+A_{4}\right)\right)<1 .
$$

Then $\mathrm{T}$ has a fixed point in $\left(-\infty, \mathrm{y}_{0}\right]$.

Proof. Let $y_{n}=T^{n} y_{0}$ for each $n$. Note that $T$ is nondecreasing on $\left(-\infty, y_{0}\right]$, then it follows from $T y_{0} \preceq y_{0}$ that

$$
y_{n+1} \preceq y_{n} \preceq \cdots \preceq y_{1} \preceq y_{0}, \quad \forall n .
$$

Set $\bar{A}=\left(I-A_{3}-A_{4}\right)^{-1}\left(A_{1}+A_{2}+A_{4}\right)$, then $r(\bar{A})<1$ by (2.13). In analogy to (2.7), by (1.1) and (2.14), we obtain

$$
\begin{aligned}
\theta \preceq y_{n}-y_{m}=\sum_{i=n}^{m-1}\left(y_{i}-y_{i+1}\right) & \preceq \sum_{i=n}^{m-1} \bar{A}^{i}\left(y_{0}-y_{1}\right) \\
& =\bar{A}^{n} \sum_{i=0}^{m-1} \bar{A}^{i}\left(y_{0}-y_{1}\right) \preceq \bar{A}^{n}(I-\bar{A})^{-1}\left(y_{0}-y_{1}\right), \quad \forall m>n .
\end{aligned}
$$

Taking $B=\bar{A}$ and $u=(I-\bar{A})^{-1}\left(x_{1}-x_{0}\right)$ in (2.3), it follows that

$$
\overline{\mathrm{A}}^{\mathrm{n}}(\mathrm{I}-\overline{\mathrm{A}})^{-1}\left(\mathrm{x}_{1}-\mathrm{x}_{0}\right) \stackrel{w}{\rightarrow} \theta(\mathrm{n} \rightarrow \infty),
$$


which together with (2.15) and Lemma 1.4 (iii) implies that

$$
\mathrm{y}_{\mathrm{n}}-\mathrm{y}_{\mathrm{m}} \stackrel{w}{\rightarrow} \theta(\mathrm{m}>\mathrm{n} \rightarrow \infty) .
$$

Thus by the Picard-completeness of $T$ at $y_{0}$, there exists $y^{*} \in E$ such that

$$
y_{n} \stackrel{w}{\rightarrow} y^{*}
$$

In analogy to (2.9), (2.10), and (2), we obtain

$$
T y^{*} \preceq y^{*} \preceq y_{n}, n=0,1,2,3, \cdots .
$$

By (1.1), (2.14), and (2.18),

$$
\begin{aligned}
\theta \preceq y_{n+1}-T y^{*} & =T y_{n}-T x^{*} \\
& \preceq A_{1}\left(y_{n}-y^{*}\right)+A_{2}\left(y_{n}-y_{n+1}\right)+A_{3}\left(y^{*}-T y^{*}\right)+A_{4}\left(y_{n}-T y^{*}\right)+A_{5}\left(y_{n+1}-y^{*}\right), \quad \forall n .
\end{aligned}
$$

Letting $n \rightarrow \infty$ in the above inequality, by (2.16), (2.17), Lemma 1.4 (iii), and Lemma 1.10 we get

$$
\theta \preceq y^{*}-T y^{*} \preceq A_{3}\left(y^{*}-T y^{*}\right)+A_{4}\left(y^{*}-T y^{*}\right),
$$

and so

$$
\left(I-A_{2}-A_{5}\right)\left(y^{*}-T^{*} y^{*}\right) \preceq \theta .
$$

Acting the above inequality with $\left(I-A_{2}-A_{5}\right)^{-1}$, we have $\theta \preceq y^{*}-T y^{*} \preceq \theta$, i.e., $y^{*}=T y^{*}$.

Remark 2.3. Theorems 2.1 and 2.2 are still valid without assumption of the Picard-completeness of T at the expense that $E$ is $\mathcal{w}$-complete by Remark 1.8 .

Proposition 2.4. Let $\mathrm{P}$ be a cone of a normed vector space $(\mathrm{E},\|\cdot\|)$ and $\mathrm{A}_{i}: \mathrm{P} \rightarrow \mathrm{P}(\mathrm{i}=1,2,3,4,5)$ be linear bounded mappings. If one of the following conditions is satisfied:

$\left(H_{1}\right) A_{i}(i=1,2,3,4,5)$ are mutually commutative (i.e., $A_{i} A_{j}=A_{j} A_{i}$ for each $\left.1 \leqslant i, j \leqslant 5\right), r\left(A_{4}\right)=r\left(A_{5}\right)$ and $\sum_{i=1}^{5} r\left(A_{i}\right)<1$;

$\left(H_{2}\right) A_{i}(i=1,2,3,4,5)$ are mutually commutative, there exists $\varepsilon \geqslant 0$ such that $r\left(A_{4}\right)-r\left(A_{5}\right)>\varepsilon$ and $\sum_{i=1}^{5} r\left(A_{i}\right)=1+\varepsilon$,

then $A_{i}(i=1,2,3,4,5)$ satisfy the condition (2.1).

Proof. Suppose that $\left(\mathrm{H}_{1}\right)$ is satisfied. Since $A_{i}(i=1,2,3,4,5)$ are mutually commutative, it follows from $\left(\mathrm{H}_{1}\right)$ that $r\left(A_{2}+A_{5}\right) \leqslant r\left(A_{2}\right)+r\left(A_{5}\right)<1$ and

$$
\begin{aligned}
r\left(\left(I-A_{2}-A_{5}\right)^{-1}\left(A_{1}+A_{3}+A_{5}\right)\right) \leqslant \frac{r\left(A_{1}+A_{3}+A_{5}\right)}{1-r\left(A_{2}+A_{5}\right)} & \leqslant \frac{r\left(A_{1}\right)+r\left(A_{3}\right)+r\left(A_{5}\right)}{1-r\left(A_{2}\right)-r\left(A_{5}\right)} \\
& =\frac{r\left(A_{1}\right)+r\left(A_{3}\right)+r\left(A_{4}\right)}{1-r\left(A_{2}\right)-r\left(A_{5}\right)}<1,
\end{aligned}
$$

and hence (2.1) holds.

Suppose that $\left(\mathrm{H}_{2}\right)$ is satisfied. Since $A_{i}(i=1,2,3,4,5)$ are mutually commutative, it follows from $\left(\mathrm{H}_{2}\right)$ that

$$
r\left(A_{2}+A_{5}\right) \leqslant r\left(A_{2}\right)+r\left(A_{5}\right) \leqslant \sum_{i=1}^{3} r\left(A_{i}\right)+2 r\left(A_{5}\right)<\sum_{i=1}^{3} r\left(A_{i}\right)+\left(r\left(A_{4}\right)-\varepsilon+r\left(A_{5}\right) \leqslant 1,\right.
$$

and

$$
r\left(\left(I-A_{2}-A_{5}\right)^{-1}\left(A_{1}+A_{3}+A_{5}\right)\right) \leqslant \frac{r\left(A_{1}+A_{3}+A_{5}\right)}{1-r\left(A_{2}+A_{5}\right)} \leqslant \frac{r\left(A_{1}\right)+r\left(A_{3}\right)+r\left(A_{5}\right)}{1-r\left(A_{2}\right)-r\left(A_{5}\right)}<1,
$$

and hence (2.1) holds. 
In analogy to Proposition 2.4, we have the following result.

Proposition 2.5. Let $\mathrm{P}$ be a cone of a normed vector space $(\mathrm{E},\|\cdot\|)$ and $\mathrm{A}_{i}: \mathrm{P} \rightarrow \mathrm{P}(\mathrm{i}=1,2,3,4,5)$ be linear bounded mappings. If either $\left(\mathrm{H}_{1}\right)$, or the following condition is satisfied:

$\left(H_{3}\right) A_{i}(i=1,2,3,4,5)$ are mutually commutative, there exists $\varepsilon \geqslant 0$ such that $r\left(A_{5}\right)-r\left(A_{4}\right)>\varepsilon$ and $\sum_{i=1}^{5} r\left(A_{i}\right)=1+\varepsilon$,

then $A_{i}(i=1,2,3,4,5)$ satisfy condition (2.13).

By Remark 1.8, Propositions 2.4 and 2.5, we have the following two corollaries corresponding to Theorems 2.1 and 2.2.

Corollary 2.6. Let $\mathrm{P}$ be a solid cone of a normed vector space $(\mathrm{E},\|\cdot\|), \mathrm{x}_{0} \in \mathrm{E}$ and $\mathrm{T}: \mathrm{D}=\left[\mathrm{x}_{0},+\infty\right) \rightarrow \mathrm{E} a$ nondecreasing order-Ćirić-Lipschitz mapping restricted with linear bounded mappings $A_{i}: P \rightarrow P(i=1,2,3,4,5)$. Assume that $\mathrm{x}_{0} \preceq \mathrm{T} \mathrm{x}_{0}$ and $\left(\mathrm{H}_{2}\right)$ is satisfied. If $\mathrm{E}$ is $\mathrm{w}$-complete, or $\mathrm{T}$ is Picard-complete at $\mathrm{x}_{0}$, then $\mathrm{T}$ has a fixed point in $\left[\mathrm{x}_{0},+\infty\right)$.

Corollary 2.7. Let $\mathrm{P}$ be a solid cone of a $w$-complete normed vector space $(\mathrm{E},\|\cdot\|), \mathrm{y}_{0} \in \mathrm{E}$ and $\mathrm{T}: \mathrm{D}=\left(-\infty, \mathrm{y}_{0}\right] \rightarrow$ $\mathrm{E}$ a nondecreasing order-Ćirić-Lipschitz mapping restricted with linear bounded mappings $\mathrm{A}_{i}: \mathrm{P} \rightarrow \mathrm{P}(\mathrm{i}=$ $1,2,3,4,5)$. Assume that $\mathrm{T}_{0} \preceq \mathrm{y}_{0}$ and $\left(\mathrm{H}_{3}\right)$ is satisfied. If $\mathrm{E}$ is $w$-complete, or $\mathrm{T}$ is Picard-complete at $\mathrm{y}_{0}$, then $\mathrm{T}$ has a fixed point in $\left(-\infty, y_{0}\right]$.

In particular when $A_{i}, B_{i}(i=1,2,3,4,5)$ are nonnegative real numbers, we have the following two fixed point results.

Corollary 2.8. Let $\mathrm{P}$ be a solid cone of a normed vector space $(\mathrm{E},\|\cdot\|), \mathrm{x}_{0} \in \mathrm{E}$ and $\mathrm{T}: \mathrm{D}=\left[\mathrm{x}_{0},+\infty\right) \rightarrow \mathrm{E} a$ nondecreasing order-Ćirić-Lipschitz mapping restricted with nonnegative real numbers $A_{i} \geqslant 0(i=1,2,3,4,5)$. Assume that $\mathrm{x}_{0} \preceq \mathrm{T} \mathrm{x}_{0}$ and the following condition is satisfied:

$\left(\mathrm{H}_{2}^{\prime}\right)$ there exists $\varepsilon \geqslant 0$ such that $\sum_{i=1}^{5} A_{i}=1+\varepsilon$ and $A_{4}-A_{5}>\varepsilon$.

If $\mathrm{E}$ is $w$-complete, or $\mathrm{T}$ is Picard-complete at $\mathrm{x}_{0}$, then $\mathrm{T}$ has a fixed point in $\left[\mathrm{x}_{0},+\infty\right)$.

Corollary 2.9. Let $\mathrm{P}$ be a solid cone of a normed vector space $(\mathrm{E},\|\cdot\|), \mathrm{y}_{0} \in \mathrm{E}$ and $\mathrm{T}: \mathrm{D}=\left(-\infty, \mathrm{y}_{0}\right] \rightarrow \mathrm{E} a$ nondecreasing order-Ćirić-Lipschitz mapping restricted with nonnegative real numbers $A_{i} \geqslant 0(i=1,2,3,4,5)$. Assume that $\mathrm{T} \mathrm{y}_{0} \preceq \mathrm{y}_{0}$ and the following condition is satisfied:

$\left(\mathrm{H}_{3}^{\prime}\right)$ there exists $\varepsilon \geqslant 0$ such that $\sum_{i=1}^{5} A_{i}=1+\varepsilon$ and $A_{5}-A_{4}>\varepsilon$.

If $\mathrm{E}$ is $w$-complete, or $\mathrm{T}$ is Picard-complete at $\mathrm{y}_{0}$, then $\mathrm{T}$ has a fixed point in $\left(-\infty, \mathrm{y}_{0}\right]$.

Remark 2.10. Corollaries 2.8 and 2.9 are still valid in Hausdorff topological vector spaces.

Remark 2.11. In Corollaries 2.6-2.9, the sum $\sum_{i=1}^{5} r\left(A_{i}\right)$ or $\sum_{i=1}^{5} A_{i}$ may be even greater than or equal to 1. Therefore, even in the setting of normal cones, Corollaries 2.6-2.9 are still new.

In particular when $P$ is normal cone of a Banach space $(E,\|\cdot\|)$, we have the following two corollaries by Proposition 1.6 and Remark 1.8.

Corollary 2.12. Let $\mathrm{P}$ be a normal cone of a Banach space $(\mathrm{E},\|\cdot\|), \mathrm{x}_{0} \in \mathrm{E}$ and $\mathrm{T}: \mathrm{D}=\left[\mathrm{x}_{0},+\infty\right) \rightarrow \mathrm{E} a$ nondecreasing order-Ćirić-Lipschitz mapping restricted with nonnegative real numbers $A_{i} \geqslant 0(i=1,2,3,4,5)$. Assume that $\mathrm{x}_{0} \preceq \mathrm{T} \mathrm{x}_{0}$ and $\left(\mathrm{H}_{2}^{\prime}\right)$ is satisfied. Then $\mathrm{T}$ has a fixed point in $\left[\mathrm{x}_{0},+\infty\right)$.

Corollary 2.13. Let $\mathrm{P}$ be a normal cone of a Banach space $(\mathrm{E},\|\cdot\|), \mathrm{y}_{0} \in \mathrm{E}$ and $\mathrm{T}: \mathrm{D}=\left(-\infty, \mathrm{y}_{0}\right] \rightarrow \mathrm{E} a$ nondecreasing order-Ćirić-Lipschitz mapping restricted with nonnegative real numbers $A_{i} \geqslant 0(i=1,2,3,4,5)$. Assume that $\mathrm{T} \mathrm{y}_{0} \preceq \mathrm{y}_{0}$ and $\left(\mathrm{H}_{3}^{\prime}\right)$ is satisfied. Then $\mathrm{T}$ has a fixed point in $\left(-\infty, \mathrm{y}_{0}\right]$. 
Example 2.14. Let $E=\{1,2,3\}$ be endowed with the usual norm $|\cdot|$ and $P=\{x \in \mathbb{R}: x \geqslant 0\}$. Then $(E,|\cdot|)$ is a Banach space and $\mathrm{P}$ is a normal cone of $\mathrm{E}$ and the partial order $\preceq$ induced by $\mathrm{P}$ is the usual total order $\leqslant$

Let $\mathrm{T} 1=\mathrm{T} 2=1$ and $\mathrm{T} 3=2$, and set $\mathrm{x}_{0}=1$. Clearly, $\mathrm{x}_{0} \preceq \mathrm{T} \mathrm{x}_{0},\left[\mathrm{x}_{0},+\infty\right)=\mathrm{E}$ and $\mathrm{T}:\left[\mathrm{x}_{0},+\infty\right) \rightarrow \mathrm{E}$ is nondecreasing. Note that $\mathrm{T} 1-\mathrm{T} 1=\mathrm{T} 2-\mathrm{T} 2=\mathrm{T} 3-\mathrm{T} 3=\mathrm{T} 2-\mathrm{T} 1=0$ and $\mathrm{T} 3-\mathrm{T} 1=\mathrm{T} 3-\mathrm{T} 2=1$, then it suffices to verify that (1.1) is satisfied with $x=3, y=1$ and $x=3, y=2$. Set $B_{i}=0(i=1,2,3,4,5)$, $A_{1}=A_{2}=A_{3}=A_{5}=\frac{1}{6}$ and $A_{4} \geqslant \frac{1}{3}$. Clearly, $\sum_{i=1}^{5} A_{i} \geqslant 1$ but $\left(H_{2}^{\prime}\right)$ is satisfied with $\varepsilon=A_{4}-\frac{1}{3}$ since $A_{4}-A_{5}=A_{4}-\frac{1}{6}>\varepsilon$. Direct calculation gives that

$$
\begin{aligned}
0 & <\mathrm{T} 3-\mathrm{T} 1=1<\frac{4}{3} \leqslant \frac{2}{3}+2 \mathrm{~A}_{4}=2 \mathrm{~A}_{1}+\mathrm{A}_{2}+2 \mathrm{~A}_{4}+\mathrm{A}_{5} \\
& =\mathrm{A}_{1}(1-3)_{+}+\mathrm{A}_{2}(3-\mathrm{T} 3)_{+}+\mathrm{A}_{3}(1-\mathrm{T} 1)_{+}+\mathrm{A}_{4}(3-\mathrm{T} 1)_{+}+\mathrm{A}_{5}(1-\mathrm{T} 3)_{+}, \\
0 & <\mathrm{T} 3-\mathrm{T} 2=1<\frac{7}{6} \leqslant \mathrm{~A}_{1}+\mathrm{A}_{2}+\mathrm{A}_{3}+2 \mathrm{~A}_{4} \\
& =\mathrm{A}_{1}(2-3)_{+}+\mathrm{A}_{2}(3-\mathrm{T} 3)_{+}+\mathrm{A}_{3}(2-\mathrm{T} 2)_{+}+\mathrm{A}_{4}(3-\mathrm{T} 2)_{+}+\mathrm{A}_{5}(2-\mathrm{T} 3)_{+} .
\end{aligned}
$$

Thus $T$ has a fixed point in $E$ by Corollary 2.12 (in fact, $x=1$ is a fixed point of $T$ ).

However, for each $A \in[0,1)$ we get $T 3-T 2=1>A=A(3-2)$, and hence none of the results of $[6,8,10,11]$ concerned with order-Lipschitz mappings is applicable here. On the other hand, for each $q, r, s, t \geqslant 0$ with $q+r+s+2 t<1$, we have

$$
\mathrm{q}|3-2|+\mathrm{r}|3-\mathrm{T} 3|+\mathrm{s}|2-\mathrm{T} 2|+\mathrm{t}(|3-\mathrm{T} 2|+|2-\mathrm{T} 3|)=\mathrm{q}+\mathrm{r}+\mathrm{s}+2 \mathrm{t}<1=|\mathrm{T} 3-\mathrm{T} 2|,
$$

i.e., $T$ is not a Ćirić's contraction and hence none of the results in $[1-3,7]$ is applicable here.

Now we come to consider the unique existence of fixed points of order-Ćirić-Lipschitz mappings.

Theorem 2.15. Let $\mathrm{P}$ be a solid cone of a normed vector space $(\mathrm{E},\|\cdot\|), \mathrm{x}_{0}, \mathrm{y}_{0} \in \mathrm{E}$ with $\mathrm{x}_{0} \preceq \mathrm{y}_{0}$ and $\mathrm{T}: \mathrm{D}=$ $\left[\mathrm{x}_{0}, \mathrm{y}_{0}\right] \rightarrow \mathrm{E}$ a nondecreasing order-Ćirić-Lipschitz mapping restricted with linear bounded mappings $\mathrm{A}_{i}: \mathrm{P} \rightarrow$ $\mathrm{P}(\mathrm{i}=1,2,3,4,5)$. Assume that $\mathrm{x}_{0} \preceq \mathrm{T} \mathrm{x}_{0}, \mathrm{~T} \mathrm{y}_{0} \preceq \mathrm{y}_{0}, \mathrm{~T}$ is Picard-complete at $\mathrm{x}_{0}$ and $\mathrm{y}_{0}$, and $\left(H_{1}\right)$ is satisfied. Then $\mathrm{T}$ has a unique fixed point $\mathrm{x}^{*} \in\left[\mathrm{x}_{0}, \mathrm{y}_{0}\right]$, and for each $z_{0} \in\left[\mathrm{x}_{0}, \mathrm{y}_{0}\right], z_{\mathrm{n}} \stackrel{w}{\rightarrow} x^{*}$, where $z_{\mathrm{n}}=\mathrm{O}\left(\mathrm{T}, z_{0}\right)$.

Proof. The existence of fixed points immediately follows from Theorems 2.1 and 2.2 and Propositions 2.4 and 2.5. Note that $T$ is nondecreasing on $\left[x_{0}, y_{0}\right]$, thus it follows from $x_{0} \preceq T x_{0}, T y_{0} \preceq y_{0}$ that

$$
x_{0} \preceq x_{1} \preceq \cdots \preceq x_{n} \preceq \cdots \preceq y_{n} \preceq \cdots \preceq y_{1} \preceq y_{0}, \quad \forall n .
$$

Letting $n \rightarrow \infty$ in (2.19), by (2.9), (2.17), and Lemma 1.4 (ii), we get

$$
x^{*} \preceq y^{*} .
$$

Thus by (1.1) and (2.19),

$$
\begin{aligned}
\theta & \preceq y_{n+1}-x_{n+1} \\
& =T y_{n}-T x_{n} \\
& \preceq A_{1}\left(y_{n}-x_{n}\right)+A_{2}\left(y_{n}-y_{n+1}\right)+A_{3}\left(x_{n+1}-x_{n}\right)+A_{4}\left(y_{n}-x_{n+1}\right)+A_{5}\left(y_{n+1}-x_{n}\right), \quad \forall n .
\end{aligned}
$$

Letting $n \rightarrow \infty$ in (2.21), by (2.8), (2.9), (2.17), (2.17), (2.20), Lemma 1.4 (iii), and Lemma 1.10, we get

$$
\theta \preceq y^{*}-x^{*} \preceq A_{1}\left(y^{*}-x^{*}\right)+A_{4}\left(y^{*}-x^{*}\right)+A_{5}\left(y^{*}-x^{*}\right),
$$

and so

$$
\left(I-A_{1}-A_{4}-A_{5}\right)\left(y^{*}-x^{*}\right) \preceq \theta .
$$


Note that $\left(I-A_{1}-A_{4}-A_{5}\right)^{-1}: P \rightarrow P$ is a linear bounded mapping by $\sum_{i=1}^{5} r\left(A_{i}\right)<1$ and taking $B=A_{1}+A_{4}+A_{5}$ in (2.2). Acting (2.22) with $\left(I-A_{1}-A_{4}-A_{5}\right)^{-1}$, we obtain $y^{*}=x^{*}$.

For each $z_{0} \in\left[x_{0}, y_{0}\right]$, we have

$$
x_{\mathrm{n}} \preceq z_{\mathrm{n}} \preceq y_{\mathrm{n}},
$$

which together with (2.9), (2.17), Lemma 1.4 (iii), and $x^{*}=y^{*}$ implies that $z_{\mathfrak{n}} \stackrel{w}{\rightarrow} x^{*}$.

Suppose that $x \in\left[x_{0}, y_{0}\right]$ is another fixed point of T. Clearly, $T^{n} x=x$ for each $n$ and $T^{n} x \stackrel{w}{\rightarrow} x^{*}$. Then by Lemma 1.4 (i), $x=x^{*}$. This shows $x^{*}$ is the unique fixed point of $T$ in $\left[x_{0}, y_{0}\right]$.

Remark 2.16. Theorem 1.2 is a particular case of Theorem 2.15 in Banach algebras with $A_{1} \in P$ and $A_{i}=\theta(i=2,3,4,5)$.

\section{Acknowledgment}

The work was supported by the National Natural Science Foundation of China $(11161022,11561026$, 71462015), the Natural Science Foundation of Jiangxi Province (20142BCB23013, 20143ACB21012, 20151BAB201003, 20151BAB201023), and the Natural Science Foundation of Jiangxi Provincial Education Department (KJLD14034, GJJ150479).

\section{References}

[1] S. Banach, Sur les operations dans les ensembles abstraits et leur application aux equations integrales, Fundam. Math., 3 (1922), 133-181.1, 2.14

[2] S. K. Chatterjea, Fixed point theorems, C. R. Acad. Bulgare Sci., 25 (1972), 727-730.1

[3] L. B. Ćirić, Generalized contractions and fixed-point theorems, Publ. Inst. Math., 12 (1971), 19-26.1, 2.14

[4] K. Deimling, Nonlinear functional analysis, Springer-Verlag, Berlin, (1985). 1

[5] S. Jiang, Z. Li, Extensions of Banach contraction principle to partial cone metric spaces over a non-normal solid cone, Fixed Point Theory Appl., 2013 (2013), 9 pages.1.3, 1.5

[6] S. Jiang, Z. Li, Fixed point theorems of order-Lipschitz mappings in Banach algebras, Fixed Point Theory Appl., 2016 (2016), 10 pages. $1,1.2,1.3,1.4,1.7,2.14$

[7] R. Kannan, Some results on fixed points, Bull. Calcutta Math. Soc., 60 (1968), 71-76.1, 2.14

[8] M. A. Krasnosel'skiǔ, P. P. Zabreǐko, Geometrical methods of nonlinear analysis, Springer-Verlag, Berlin, (1984). 1, 2.14

[9] Z. Li, S. Jiang, Common fixed point theorems of contractions in partial cone metric spaces over nonnormal cones, Abstr. Appl. Anal., 2014 (2014), 8 pages.1.3, 1.4, 1.5

[10] J.-X. Sun, Iterative solutions of nonlinear operator, (Chinese) J. Engin. Math., 6 (1989), 12-17. 1, 2.14

[11] X. Y. Zhang, J. X. Sun, Existence and uniqueness of solutions for a class of nonlinear operator equations and its applications, (Chinese) Acta Math. Scientia, 25 (2005), 846-851.1, 2.14 